\title{
ESTUDIO CARIOLÓGICO DEL GÉNERO PARONYCHIA MILLER (CARYOPHYLLACEAE) EN ANDALUCÍA, (ESPAÑA)
}

\author{
Juan Carlos DIOSDADO y Julio PASTOR
}

\begin{abstract}
RESUMEN. Estudio cariológico del género Paronychia Miller (Caryophyllaceae) en Andalucía, (España). Se realiza el estudio cariológico de seis especies pertenecientes al género Paronychia en la Península Ibérica. Se han encontrado tres números básicos $(x=7,8$ y 9). Se incluyen cariogramas de todas las especies, siendo nuevo el dato cariológico de $P$. suffruticosa, especie endémica del sureste peninsular.
\end{abstract}

Palabras clave. Cariología, Paronychia, Caryophyllaceae, Península Ibérica.

SUMMARY. Karyological study of the genus Paronychia Miller (Caryophyllaceae) from Andalusia, (Spain). A karyological study of six species of the genus Paronychia from the Iberian Peninsula has been made. The basic numbers $x=7,8$ and 9 have been found. Caryograms are given in all the species, and for the endemic species $P$. suffruticosa is reported for the first time.

Key words. Karyology, Paronychia, Caryophyllaceae, Iberian Peninsula.

\section{INTRODUCCIÓN}

El género Paronychia está formado por más de un centenar de especies repartidas por América, Europa, Asia y Norte de Africa. La región mediterránea es el principal centro de distribución, presentando dos áreas donde se localizan cerca del $50 \%$ de las especies: por un lado la zona de los Balcanes, Turquía y Oriente Próximo y por otro el área comprendida por el Sur de España y el Norte de África (Chaudhri, 1968).

Desde el punto de vista cariológico, este género ha sido muy poco estudiado. Küpfer (1974) realizó un estudio citotaxonómico de P. kapela (Hacq.) Kerner estableciendo los cariotipos de las distintas subespecies. El resto de los estudios se refieren a números cromosómicos aislados de una docena de especies. Salvo el caso de P. kapela (Küpfer, 1. c.) no se había aportado información alguna sobre tamaño, asimetría o morfología de los cromosomas de Paronychia. En el presente trabajo se indican por primera vez estos datos cariológicos para 6 especies del género distribuidas por el Sur de la España peninsular.

\section{MATERIAL Y MÉTODOS}

Las observaciones de las meiosis se realizaron a partir de botones florales fijados en el 
campo con líquido de Farmer, alcohol etílicocloroformo-ácido acético en proporción 6:3:1 (Löve \& Löve, 1975), durante 24 horas, tras las cuales se pasaron a alcohol etílico al $70 \%$ donde se conservaron hasta su tinción. Esta se realizó con carmín clorhídrico etílico (Snow, 1963) durante 72 horas. Debido a la existencia de brácteas desarrolladas en la mayoría de las especies estudiadas, que impidieron la correcta fijación, la observación de los cromosomas en meiosis ha sido laboriosa y poco fructífera.

Para el estudio de mitosis se utilizaron meristemos radicales obtenidos a partir de semillas, los cuales fueron tratados con 8hidroxiquinoleina $0,002 \mathrm{M}$ (Tjio \& Levan, 1950) durante 4 horas y fijados en Carnoy (Löve \& Löve, 1975) durante un mínimo de 24 horas para pasarlos posteriormente a alcohol etílico al $70 \%$. La tinción se efectuó con carmín-alcohólico-clorhídrico (Snow, 1963), manteniéndose en dicho colorante durante 3 ó 4 días. Seguidamente se procedió a su preparación, que se ha realizado por aplastamiento en ácido acético al $45 \%$.

Para indicar la morfología de los cromosomas se ha seguido la terminología de Levan \& al. (1964). El tamaño aparente de los mismos y el grado de asimetría de los cariotipos se indica de acuerdo con Stebbins (1938 y 1971).

El número de individuos estudiados en cada población varió de 5 a 10 . A continuación se indican las localidades estudiadas para cada especie. Los ejemplares utilizados se indican por medio del número de pliego con que se conservan en el Herbario del Departamento de Biología Vegetal y Ecología de la Universidad de Sevilla (SEV).

\section{MATERIAL ESTUDIADO}

\section{P. suffuticosa (L.) DC.}

GRANADA: Lacalahorra, subida al Puerto de la Ragüa, 1600 m.s.m., 20-V-1992, Diosdado (SEV 135501). ALMERIA: Cabo de Gata, 21-V-1992,
Diosdado (SEV 135500). Entre los Escullos y El Mirador de la Amatista, 18-VIII-1993, Diosdado (SEV 135499).

\section{P. echinulata A. O. Chater.}

CADIZ: Tarifa, 1-VII-1993, Diosdado (SEV 135498). Chiclana, 5-VIII-1993, Diosdado (SEV 135497).

P. polygonifolia (Vill.) DC.

GRANADA: Sierra Nevada, Subida al Veleta, 2800 m.s.m., 16-VIII-1993, Diosdado (SEV 135496). Sierra Nevada, entre el Veleta y Capileira, 16-VIII-1993, Diosdado (SEV 135495).

P. argentea Lam.

ALMERIA: Cabo de Gata, 21-V-1992, Diosdado (SEV 135494). Santa María de Nieva, 21-V-1992, Diosdado (SEV 135493). Los Escullos, 17-VIII-1993, Diosdado (SEV 135492). CADIZ, Tarifa, 4-VIII-1993, Diosdado (SEV 135491). Barbate, 4-VIII-1993, Diosdado (SEV 135490). Sanlúcar de Barrameda, 30-IX-1993, Juan \& Pastor (SEV 135489). Bolonia, 1-VII-1993, Diosdado (SEV 135488). HUELVA, Punta Umbría, 29-VI1992, Diosdado (SEV 135487). JAEN, Sierra de Cazorla, Puerto de las Palomas, 16-VI-1992, Diosdado (SEV 135486).

P. aretioides Pourret ex DC.

GRANADA: Huéscar, salida hacia Puebla de D. Fadrique, 21-V-1992, Diosdado (SEV 135485). ALMERIA, Puerto de María, 21-V-1992, Diosdado (SEV 135484).

\section{P. capitata (L.) Lam.}

ALMERIA: Santa María de Nieva, 21-V-1992, Diosdado (SEV 135483). Puerto de Santa María de Nieva, 21-V-1992, Diosdado (SEV 135482). Rambla Honda, 20-V-1992, Diosdado (SEV 135481). GRANADA: Lacalahorra, 20-V-1992, Diosdado (SEV 135480). JAEN: Sierra Mágina, Torres, 16-VI-1992, Diosdado (SEV 135479).

\section{RESULTADOS}

\section{Subgénero Paronychia}

P. suffruticosa (L.) DC., in Lam., Encyclop. 5: 25 (1804) 
En el material procedente de Granada (SEV 135501) se encontró el número gamético $\mathrm{n}=8$ y en las dos poblaciones de Almería se observó el número somático $2 \mathrm{n}=16$.

Las meiosis presentaban la formación regular de 8 bivalentes. Por otro lado, en las metafases mitóticas estudiadas, el tamaño aparente de los cromosomas varió entre 2.47 y $4.30 \mu \mathrm{m}$ (medianamente pequeños). El tipo de asimetría es $3 \mathrm{~A}$ y el cariograma presenta, en la población de Los Escullos, la fórmula: $4 m+4 s m+6 s t+2 t$ (fig. 1, A).

De acuerdo con la bibliografía consulta$\mathrm{da}$, parecen ser los primeros datos referentes a la cariología de esta especie endémica del sureste peninsular.

P. echinulata A. O. Chater in Feddes Repert. Spec. Nov. Regni Veg. 69: 52 (1964)

En las dos poblaciones estudiadas de Cádiz se encontró el número somático $2 n=14$, el cual concuerda con el aportado por Chaudhri (1968) en material de origen desconocido. Sin embargo, Blackburn \& Morton (1957) indicaron $2 n=28$ con plantas procedentes de Portugal y Chaudhri (1968) observó una población con $2 n=24$.

Parecen ser los primeros recuentos realizados con material español.

En el cariotipo de las muestras estudiadas con material andaluz, el tamaño aparente de los cromosomas varió entre 1.82 y $3.87 \mu \mathrm{m}$ (entre pequeños y medianamente pequeños). El tipo de asimetría es $3 \mathrm{~A}$. El cariograma de $P$. echinulata presenta la fórmula $4 \mathrm{~m}+4 \mathrm{sm}+6 \mathrm{st}$ (fig. 1, B).

P. polygonifolia (Vill.) DC. in Lam. \& DC., Fl. Franç. ed. 3, 3: 403 (1805)

El número cromosómico encontrado $2 n=14$, con material procedente de Sierra Nevada, coincide con los aportados por varios autores: Blackburn \& Morton (1957) con material de Portugal, Küpfer $(1971,1974)$ para poblaciones de los Pirineos franceses y espa- ñoles, Diana Corrias \& Villa (1980) en muestras de Italia y Galland \& Küpfer (1984) para poblaciones de España y Portugal. Por otro lado, Galland $(1985,1988)$ observó, en poblaciones marroquíes, $2 \mathrm{n}=14$ para la var. velucensis Boiss. y $2 \mathrm{n}=28$ para la vàr. serratifolia Chaudhri.

En las poblaciones estudiadas, el tamaño de los cromosomas osciló entre 1.40 y $2.04 \mu \mathrm{m}$ (entre pequeños y medianamente pequeños). El cariotipo presenta en este caso una asimetría del tipo 2A. El cariograma obtenido tiene la fórmula $10 \mathrm{~m}+4 \mathrm{sm}$ (fig. $1, \mathrm{C}$ ).

\section{P. argentea Lam., Fl. Franç. 3: 230 (1779)}

En todas las poblaciones muestreadas, se ha observado $2 n=28$, lo cual confirma los recuentos de Mesquita (1953), Blackburn \& Morton (1957) y Fernandes \& Leitao (1971), todos ellos con material portugués. Asimismo, Castroviejo (1983) y Galland (1988) también encontraron este número en muestras españolas y marroquíes, respectivamente. Por otro lado, Chaudhri (1968) y Galland (1988) observaron plantas con $2 n=14$. Sin embargo, Lorenzo Andreu \& García Sanz (1950) y Löve \& Kjellqvist (1974) encontraron metafases somáticas con 36 cromosomas en varias poblaciones españolas de Zaragoza y Jaén.

El tamaño de los cromosomas en esta especie varió entre 1.6 y $3.02 \mu \mathrm{m}$ (entre pequeños y medianamente pequeños). El tipo de asimetría es $2 \mathrm{~A}$.

El cariograma obtenido en la población del Cabo de Gata (Almería) tiene la fórmula $12 m+8 s m+2$ (sm-st) $+6 s t$.

Para la muestra de Barbate (Cádiz) la fórmula del cariograma obtenido es $12 \mathrm{~m}+2(\mathrm{~m}$ $s m)+8 s m+2 s m^{\text {sat }}+2 s t+2 s t^{\text {sat }}$ (fig. 1, D).

\section{Subgénero Anoplonychia (Fenzl) Chaudhri}

P. aretioides Pourret ex DC., Prodr. 3: 371 (1828) 
El número cromosómico $2 \mathrm{n}=18$, encontrado en esta especie endémica del sureste peninsular, coincide con el aportado anteriormente por Fernández Casas (1977) para una población de Granada.

En el cariotipo de la muestra de Huéscar (Granada), el tamaño aparente de los cromosomas osciló entre 1.29 y $4.95 \mu \mathrm{m}$ (entre pequeños y medianamente pequeños). El tipo de asimetría es 2B y el cariograma tiene la fórmula $10 \mathrm{~m}+8 \mathrm{st}$ (fig. 1, E).

\section{P. capitata (L.) Lam., Fl. Franç. 3: 229 (1779)}

En todas las poblaciones estudiadas se observó el número cromosómico $2 \mathrm{n}=36$. Anteriormente sólo se conocían los datos de Blackburn \& Morton (1957) que observaron $2 \mathrm{n}=\mathrm{c} .42$ en material de Portugal y Van Loon \& Snelders (1979) que encontraron $2 n=18$ en muestras de Grecia.

De acuerdo con la bibliografía consultada son los primeros recuentos realizados con material español.

En las poblaciones andaluzas, el tamaño aparente de los cromosomas varió entre 0.97 y $1.50 \mu \mathrm{m}$ (pequeños). El tipo de asimetría encontrado es $2 \mathrm{~A}$.

El cariograma obtenido en la población de Sierra Mágina (Jaén) presenta los cromosomas en grupos de cuatro: $20 \mathrm{~m}+8(\mathrm{~m}$ sm) +4 sm+4st (fig. 1, F).

\section{DISCUSIÓN}

Desde un punto de vista cariológico, el género Paronychia se caracteriza por presentar cariotipos simétricos, cromosomas de pequeño tamaño (menores de $5 \mu \mathrm{m}$ ) y varios números básicos y niveles de ploidía.

Las metafases somáticas observadas sugieren que en Paronychia, existe un predominio de cromosomas metacéntricos con un $49.3 \%$ seguidos de los cromosomas submetacéntricos y subtelocéntricos con un $27.7 \%$ y un $21.7 \%$, respectivamente. Por último, se encuentran los cromosomas telocéntricos que sólo constituyen el $1.3 \%$. Por el alto porcentaje de cromosomas metacéntricos y submetacéntricos, que suponen un $77 \%$, los taxones del género Paronychia presentan por lo general un cariotipo bastante simétrico.

La especie con cariogramas más asimétricos es $P$. aretioides con un tipo 2B presentando la mayor diferencia de tamaño entre los cromosomas de su cariotipo. Con una asimetría del tipo 3A se hallan $P$. suffruticosa, $P$. echinulata que presentan la mayor proporción de cromosomas subtelocéntricos y telocéntricos.

Por el contrario, P. polygonifolia, P. capitata y $P$. argentea presentan los cariotipos más simétricos con un tipo $2 \mathrm{~A}$, que se caracterizan por que en sus cariogramas existe muy poca diferencia de tamaño entre todos sus cromosomas y porque los cromosomas subtelocéntricos y telocéntricos están presentes en menor proporción.

El tamaño cromosómico varió, en las especies estudiadas, entre $0.97 \mu \mathrm{m}$ del cromosoma más pequeño de $P$. capitata hasta $4.95 \mu \mathrm{m}$ del mayor cromosoma de $P$. aretioides. Por ello, atendiendo a la terminología de Stebbins (1938), varían entre pequeños y medianamente pequeños.

El subgénero Paronychia presenta, en el área estudiada, los números básicos $\mathrm{x}=7$ y 8 . En $P$. echinulata se ha observado el número básico $\mathrm{x}=7$, aunque Chaudhri (1968) también indicó $x=¿ 6$ ó 12 ? para un recuento de $2 n=24$. Ello supondría que en esta especie existe disploidía con dos números básicos $(x=7$ y ¿6 ó 12?) y distintos niveles de ploidía (2x y 4x), ya que para $P$. echinulata los números encontrados son $2 n=14,24$ y 28 . En $P$. argentea ocurre un fenómeno similar ya que se conocen los números $2 \mathrm{n}=14,28,36$ y 56 . Si aceptamos los recuentos atípicos de $2 n=36$, esto supondría la existencia de disploidía con dos números básicos $\mathrm{x}=7$ y 9 , con la presencia de 

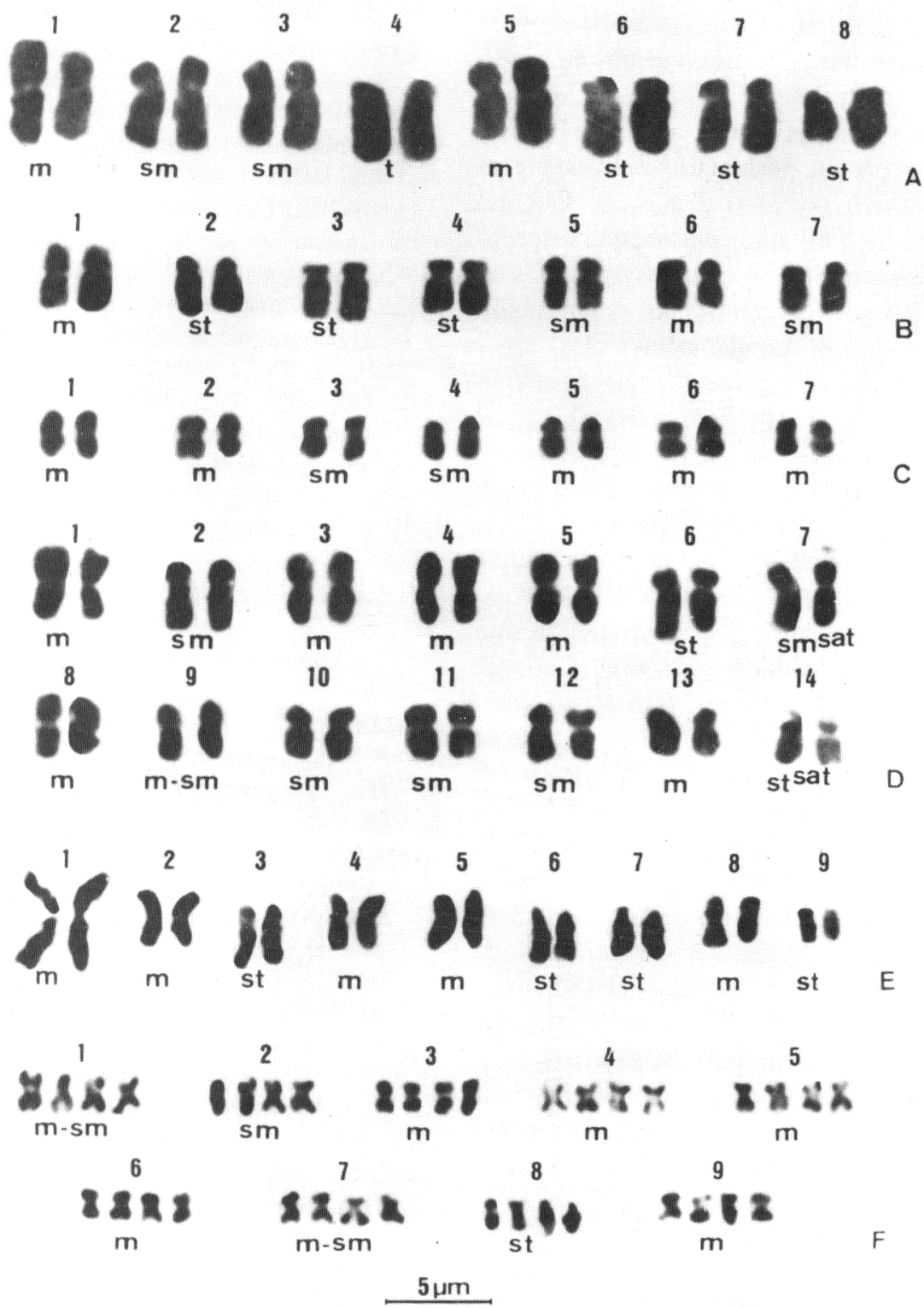

Figura 1. Cariogramas del género Paronychia. A: P. suffruticosa (SEV 135499). B: P. echinulata (SEV 135498). C: P. polygonifolia (SEV 135496). D: P. argentea (SEV 135490). E: P. aretioides (SEV 135485). F: P. capitata (SEV 135479). Caryograms of the genus Paronychia. 
diploides, tetraploides y octoploides en el caso de $x=7$ y sólo de tetraploides con $x=9$. Aunque también es posible que los recuentos de $2 n=36$ correspondan realmente a $P$. capitata, especie confundida a veces con la anterior. En este caso $P$. argentea presentaría únicamente el número básico $\mathrm{x}=7$. Las demás especies del subgénero no presentan controversia respecto del número básico. Por los datos obtenidos en este estudio, $P$. suffruticosa es un taxón diploide con $\mathrm{x}=8$. Aunque existen otras especies con este número básico, como $P$. andina A. Gray (Diers, 1961) y P. canariensis (L. fil.) Juss. (Borgen, 1969), en estas sólo se ha encontrado el nivel tetraploide $(2 \mathrm{n}=32) . P$. polygonifolia tiene número básico $\mathrm{x}=7 \mathrm{y}$ presenta dos niveles de ploidía: el nivel diploide ha sido hallado en las variedades polygonifolia y velucensis, mientras que el tetraploide sólo lo tiene la variedad serratifolia (Galland, 1988), que es endémica de Marruecos.

En el subgénero Anoplonychia sólo se ha observado el número básico $\mathrm{x}=9$, como había apuntado anteriormente Küpfer (1974) para las distintas subespecies de $P$. kapela. En $P$. aretioides, taxón endémico del sureste peninsular, se encontró el nivel diploide con número básico $\mathrm{x}=9$. Para $P$. capitata se ha observado por primera vez, en la poblaciones andaluzas, el número somático $2 n=36$. Anteriormente otros autores habían aportado $2 \mathrm{n}=18$ y c. 42 , por lo que este especie parece presentar una serie poliploide $(2 \mathrm{x}, 4 \mathrm{x}$ y $i 5 \mathrm{x}$ ?) de número básico $\mathrm{x}=9$, donde las poblaciones españolas serían tetraploides.

\section{BIBLIOGRAFÍA}

BORGEN, L. -1969- Chromosome numbers of vascular plants from the Canary Islands with special reference to the occurrence of polyploidy. Norw. J. Bot., 16: 81-121.

BLACKBURN, K. B. \& J. K. MORTON - 1957- The indicence of polyploidy in the Caryophyllaceae of Britain and of Portugal. New Phytol., 56:
334-352.

CASTROVIEJO, S. -1983- números cromosomáticos de plantas occidentales, 211-222. Anales Jard. Bot. Madrid, 39: 525-531.

CHAUDHRI, M. N. -1968- A revision of the Paronychiinae. Med. Bot. Mus. Herb. Rijks Univ. Utrech, 285: 1-297.

DIANA CORRIAS, S. \& R. VILLA -1980- Numeri cromosomici per la flora italiana: 708-713. Inform. Bot. Ital., 12: 131-134.

DIERS, L. -1961- Der Anteil an Polyploiden in den Vegetationsgürteln der Westkordillere Perus. Zeitschr. Bot., 49: 437-488.

FERNANDES, A. \& M. T. LEITAO -1971Contribuition à la connaissance cytotaxinomique des Spermatophyta du Portugal. III. Caryophyllaceae. Bol. Soc. Brot., Sér., 2, 45: 143-176.

FERNÁNDEZ CASAS, J. -1977- Recuentos cromosómicos en plantas vasculares españolas. Saussurea, 8: 33-55.

GALLAND; N. -1985- In A. Löve (ed.) IOPB Chromosome numbers reports, LXXXVII. Taxon, 34: 347-348.

GALLAND, N. -1988- Recherche sur l'origine de la flore orophile du Maroc. Étude caryologique et cytogeographique. Trav. Inst. Sci. Ser. Bot., 35, Rabat.

GALLAND, N. \& P. KÜPFER -1984- La différenciation caryologique de quelques orophytes OuestEuropéens-Maghrébins et le problème de leur mise en place. Webbia, 38: 473-490.

KÜPFER, P. -1971- Liens génétiques entre les flores alpienne et pyrénéenne. Acta Coll. Fl. Veg. Alpine et Jurass:, 167-185.

KÜPFER, P. -1974- Recherches sur les liens de parenté entre la flore orophile des Alpes et celle des Pyrénées. Boissiera, 23: 1-322.

LEVAN, A., K. FREDGA \& A. A. SANDBERG 1964- Nomenclature for centromeric position on chromosomes. Hereditas, 52: 201-220.

LOON, J. C., van \& H. M. SNELDERS -1979- In A. Löve (ed.) IOPB Chromosome number reports, LXV. Taxon, 28: 632-634.

LORENZO ANDREU, A. y P. GARCÍA SANZ 1950- Cromosomas somáticos de plantas espontáneas en la estepa de Aragón. II. Anal. Est. Exp. Aula Dei, 2: 12-20.

LÖVE, A. \& E. KJELQVIST -1974- Cytotaxonomy of Spanish plants. III. Dicotyledons: SalicaceaeRosaceae. Lagascalia, 4: 3-32. 
LÖVE, A. \& D. LÖVE -1975-Plant chromosomes. Vaduz.

MESQUITA, J. E., de -1953- Contribuiçao para o conhecimento cariológico das halofitas e psamófitas litorais. Diss. Univ. Coimbra, 1953.

SNOW, R. -1963- Alcoholic hydrochloric acidcarmine as a stain for chromosomes in squash preparations. Stain Technol., 38: 9-13.

STEBBINS, G. L. -1938-Cytological characteristics associated with the different growth habits in the dicotyledons. Amer. J. Bot., 25: 189-198.

STEBBINS, G. L. -1971- Chromosomal evolution in higher plants. London.
TJIO, J. J. \& A. LEVAN -1950- The use of oxyquinoleine in chromosome analysis. Anal. Est. Exper. Aula Dei, 2: 21-64.

Aceptado para su publicación en Febrero de 1994

Dirección de los autores. Departamento de Biología Vegetal y Ecología, Universidad de Sevilla, Apdo. 1095, 41080 Sevilla. 